\title{
Detection of Apple Scar Skin Viroid by Reverse Transcription Recombinase Polymerase Amplification Assay
}

*Corresponding author Tel: $+82-62-530-2075$

Fax: +82-62-530-2069

E-mail: jraed2@jnu.ac.kr ORCID

https://orcid.org/0000-0002-6370-8909

Received June 17, 2021 Revised June 29, 2021

Accepted June 29, 2021

\author{
Na-Kyeong Kim ${ }^{1}$, Hyo-Jeong Lee', Tae-Ho Ryu' ${ }^{1}$, In-Sook $\mathrm{Cho}^{2}$, Ho-Jong Ju' ${ }^{3}$, and \\ Rae-Dong Jeong ${ }^{1 *}$ (D) \\ ${ }^{1}$ Department of Applied Biology, Institute of Environmentally Friendly Agriculture, Chonnam National \\ University, Gwangju 61185, Korea \\ ${ }^{2}$ Horticultural and Herbal Crop Environment Division, National Institute of Horticultural and Herbal \\ Science, Rural Development Administration, Wanju 55365, Korea \\ ${ }^{3}$ Department of Agricultural Biology, College of Agriculture \& Life Sciences, Jeonbuk National University, \\ Jeonju 54896, Korea
}

\begin{abstract}
The aim of the present study was to develop a sensitive and specific detection method for the rapid detection of apple scar skin viroid (ASSVd) in apple leaves. The resulting reverse transcription recombinase polymerase amplification (RT-RPA) assay can be completed in $10 \mathrm{~min}$ at $42^{\circ} \mathrm{C}$, is 10 times more sensitive than conventional reverse transcription polymerase chain reaction, and can specifically amplify ASSVd without any cross-reactivity with other common apple viruses, including apple stem grooving virus, apple stem pitting virus, and apple chlorotic leaf spot virus. The reliability of the RT-RPA assay was assessed, and the findings suggested that it can be successfully utilized to detect ASSVd in field-collected samples. The RT-RPA assay developed in the present study provides a potentially valuable means for improving the detection of ASSVd in viroid-free certification programs, especially in resource-limited conditions.
\end{abstract}

Keywords: Apple leaves, Apple scar skin viroid, Detection, Reverse transcription recombinase polymerase amplification
Apple scar skin viroid (ASSVd), which is a member of the genus Apscaviroid (Pospiviroidae), is an economically significant pathogen of pome fruits that has been reported to cause a variety of fruit diseases (e.g., apple scar skin, apple dapple fruit, pear rusty skin, and pear dimple fruit) in Asia, Europe, and North America (Hadidi et al., 1990; Hashimoto and Koganezawa, 1987; Osaki et al., 1996). Infection by ASSVd results in intense dappling, scarring, or cracking of ripening fruit skins and causes significant economic losses to apple producers (Walia et al., 2009). ASSVd is typically transmitted through grafting and the use of contaminated pruning tools

Research in Plant Disease

pISSN 1598-2262, elSSN 2233-9191

www.online-rpd.org but, in rare cases, can also be transmitted to seeds vertically (Kim et al., 2006). Recently, a large-scale survey revealed that the infection rate of apple and pear trees in Korea was 1.7\% and 3.6\%, respectively, and ASSVd has also been detected in naturally occurring mixed infections of apple and pear trees, along with apple chlorotic leaf spot virus (ACLSV), apple stem pitting virus (ASPV), and apple stem grooving virus (ASGV) (Kim et al., 2010; Kim et al., 2019). Because of its prevalence and economic significance, ASSVd is now considered a highrisk pathogen by virus-free certification programs for fruit trees in Korea.

Viroids lack coding sequences for peptides or proteins, thereby limiting the utilization of serological approaches (Diener, 2001); hence, both reverse transcription polymerase chain reaction (RT-PCR) and TaqMan-based RT-PCR methods 
have been developed as alternative means of ASSVd detection (Heo et al., 2019; Khan et al., 2015; Kumar et al., 2014). For example, the Korean Ministry of Agriculture, Food and Rural Affairs (MAFRA) currently uses conventional RT-PCR to manage ASSVd in apple and pear orchards and to produce viroidfree fruit trees for farmers (Kim et al., 2010). However, these methods are expensive, and time-consuming, and require specialized equipment and staff. Recently, reverse transcription loop-mediated isothermal amplification (RT-LAMP) has been used to detect ASSVd in apple leaves (Lee et al., 2018). However, despite the method's robustness, simplicity, and relevance to resource-limited conditions, its application is constrained by the difficulty of primer design, relatively high temperatures required for incubation, and susceptibility to false-positive visual reactions (Panno et al., 2020).

Recombinase polymerase amplification (RPA) is an isothermal method for nucleic acid amplification and is known as a rapid, simple, cost-effective, and user-friendly diagnostic tool, owing to its ability to amplify target DNA within 15 min at constant, low temperatures $\left(25-42^{\circ} \mathrm{C}\right)$ and to its lack of prohibitive requirements, such as costly equipment (e.g., thermal cycler), high-temperature denaturation steps, or multiple primer sets (Piepenburg et al., 2006). However, few RPA-based assays have been developed for the detection of viroids (Hammond and Zhang, 2016; Lee et al., 2020). Therefore, the aim of the present study was to develop an RPAbased method for the detection of ASSVd in apple leaves.

ASSVd-infected and ASSVd-free apple leaves were col- lected from apple orchards in Jeonju city, South Korea, and stored at $-80^{\circ} \mathrm{C}$. Total RNA was then extracted using the Clear-S Total RNA Extraction Kit (InVirusTech Co., Gwangju, Korea), following the manufacturer's instructions, and RNA concentration and quality were determined using a Nanodrop spectrophotometer (BioDrop, Cambridge, UK). The ASSVd infection status of each leaf sample was determined by RT-PCR using SuPrimeScript RT-PCR Premix (Kim et al., 2010) (GeNet Bio, Daejeon, Korea), a diagnostic primer set provided by MAFRA, and the following reaction conditions: $50^{\circ} \mathrm{C}$ for $30 \mathrm{~min} ; 95^{\circ} \mathrm{C}$ for $5 \mathrm{~min} ; 35 \mathrm{cycles}$ of $95^{\circ} \mathrm{C}$ for $30 \mathrm{sec}$, $56^{\circ} \mathrm{C}$ for $60 \mathrm{sec}$, and $72^{\circ} \mathrm{C}$ for $30 \mathrm{sec}$; and $72^{\circ} \mathrm{C}$ for $10 \mathrm{~min}$. The resulting amplified fragments were visualized using gel electrophoresis on $1.5 \%$ agarose gels. The confirmed ASSVd-free and -infected samples were then used as negative and positive samples, respectively, for additional experiments.

To develop primers for the ASSVd reverse transcription recombinase polymerase amplification (RT-RPA) assay, the complete genome sequences of ASSVd isolates from Korea (LC086301, LC431517, LC276944, EU825613, KP765434, KP765429, and AF421195) and various other countries (KC110861, DQ362907, X17696, FJ974099, GQ249349, and LT577622) were obtained from GenBank, and BioEdit ver. 7.0.5.3 was used to select highly sequences. PrimedRPA (Higgins et al., 2019) and the TwistDx RPA manual (TwistDx, Ltd., Cambridge, UK) were then used to develop optimal RTRPA primer sets, and the resulting primer pair (ASSVd FP1, 5'-CCCCGCCAACGCAGATAGATAAAGAAAACGAG-3'; ASSVd
A

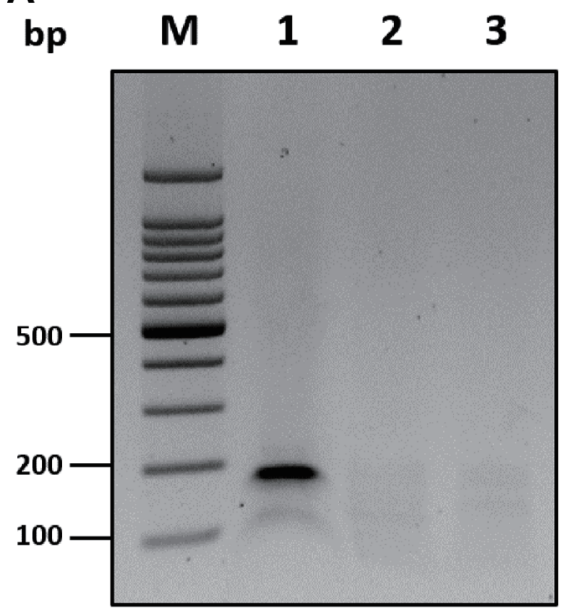

B

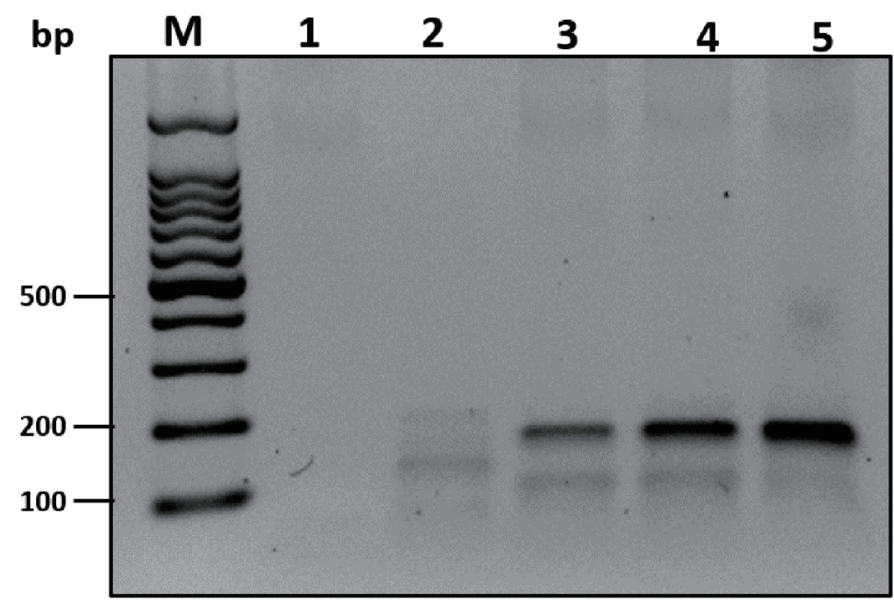

Fig. 1. Optimization of reverse transcription recombinase polymerase amplification (RT-RPA) assay for apple scar skin viroid (ASSVd) detection. (A) Gel image of ASSVd-specific RT-RPA amplicon ( 188 bp). M, marker; lane 1, ASSVd-infected RNAs; lane 2, ASSVd-free RNAs; lane 3, no-template control. (B) Effect of incubation time on ASSVd detection. Lane M, DNA marker; lanes 1-5, amplification product after 1, 5, 10, 20, and 30 min of incubation. The presented results are representative of three independent experiments. 
FP2, 5'-CCTCAACACCGTACGTTCTTGTAGGCGAACCC-3') was used to target a 188-bp fragment that spanned nucleotide positions 20 to 217 (Supplementary Fig. 1).

To optimize reaction conditions, RT-RPA assays were performed using the TwistAmp Basic RT kit (TwistDx) in 50- $\mu$ l reaction volumes, each containing $0.5 \mu \mathrm{g}$ total RNA, $29.5 \mu \mathrm{l}$ $1 \times$ rehydration buffer, and $0.24 \mu \mathrm{M}$ of each primer, and the reactions were incubated at $42^{\circ} \mathrm{C}$ for $1-30$ min using a water bath. ASSVd-infected samples were used as positive controls, and ASSVd-free samples and non-template controls were used as negative controls. The resulting amplified fragments were visualized on $2 \%$ agarose gels that contained ethidium bromide (Fig. 1A). The reaction was performed at $42^{\circ} \mathrm{C}$ for 20 min. To optimize the RT-RPA reaction time, the reaction was allowed to incubate for 1, 5, 10, 20, and 30 min, respectively. Results showed that total RNA from the ASSVd-infected apple leaves produced a clear DNA band of $\sim 188 \mathrm{bp}$ when the reaction time was only $10 \mathrm{~min}$ (Fig. 1B). The optimal conditions, $42^{\circ} \mathrm{C}$ (reaction temperature) and $10 \mathrm{~min}$ (reaction time), were used for further experiments. Three independent reaction time optimization experiments using different RNA samples were performed and similar results were obtained. The results indicated that a 10-min reaction time was sufficient for detection of ASSVd.

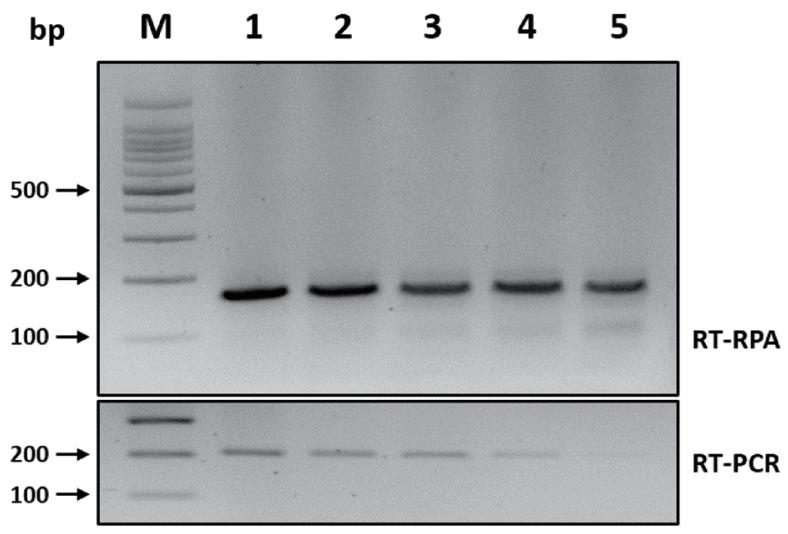

Fig. 2. Comparison of the sensitivity of reverse transcription recombinase polymerase amplification (RT-RPA) assay in the detection of apple scar skin viroid (ASSVd). The total RNAs extracted from ASSVd-infected apple leaves were used as a template to determine the detection limits of RT-RPA and reverse transcription polymerase chain reaction (RT-PCR). The reaction products (188 bp for the RT-RPA assay and 202 bp for the RT-PCR assay) were visualized on $2 \%$ agarose gels. M, DNA maker; lanes $1-5$, serial 10 -fold dilutions of RNA (ranging from $200 \mathrm{ng} / \mu \mathrm{l}$ to $20 \mathrm{pg} / \mu \mathrm{l}$ of total RNAs). Four independent reactions were performed, and similar results were observed.
To assess the sensitivity of the RT-RPA assay, 10-fold serial dilutions (starting from $200 \mathrm{ng} / \mu \mathrm{l}$ ) of total ASSVd-infected apple leaf RNA were subject to both RT-PCR and RT-RPA amplification, with three independent assays per dilution series. The results showed that ASSVd was detected in the total RNA diluted up to $20 \mathrm{pg} / \mathrm{\mu l}$ ( $10^{-4}$ dilution) by RT-RPA. However, ASSVd was detected in the total RNA diluted up to $200 \mathrm{pg} / \mu \mathrm{l}$ ( $10^{-3}$ dilution) by RT-PCR (Fig. 2). These results indicated that sensitivity of RT-RPA assay was at least 10 times higher than that of the conventional RT-PCR assay.

Meanwhile, to assess specificity, the RT-RPA and RT-PCR assays were evaluated against ASGV, ASPV, and ACLSV, which are major pathogens of apple in Korea. Previously reported primer sets for ASGV, ASPV, and ACLSV were used for RT-PCR (Kim et al., 2019). For the ASSVd assay, no cross-reaction was observed for any of the other apple viruses, which indicated that the RT-RPA assay was sufficiently specific for ASSVd (Fig. 3).

To determine reliability of the RT-RPA assay for ASSVd, 12 apple leaf samples with or without ASSVd-like symptoms were subjected to RT-PCR and RT-RPA, respectively. The results showed that ASSVd was detected by both RT-PCR

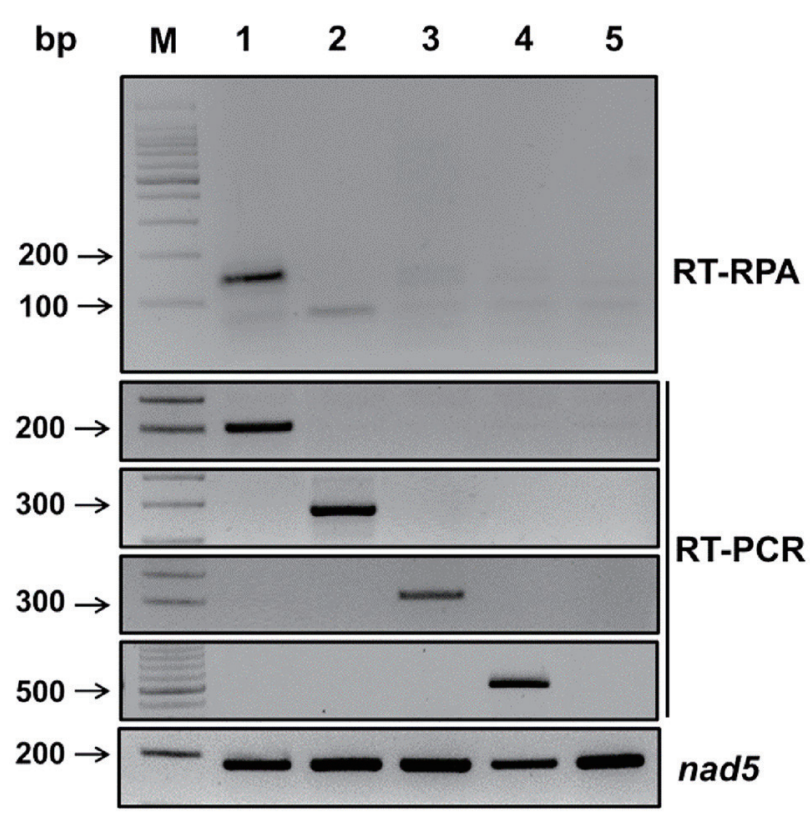

Fig. 3. Specificity of reverse transcription recombinase polymerase amplification (RT-RPA) assay for the detection of apple scar skin viroid (ASSVd) in apple leaf samples. Lane M, 1,000-bp marker; lanes 1-5, RT-RPA products amplified from ASSVd-, apple stem grooving virus (ASGV)-, apple stem pitting virus (ASPV)-, apple chlorotic leaf spot virus (ACLSV)-infected apple leaves, and healthy apple leaf sample, respectively. nad 5 was used as an internal control. RT-PCR, reverse transcription polymerase chain reaction. 


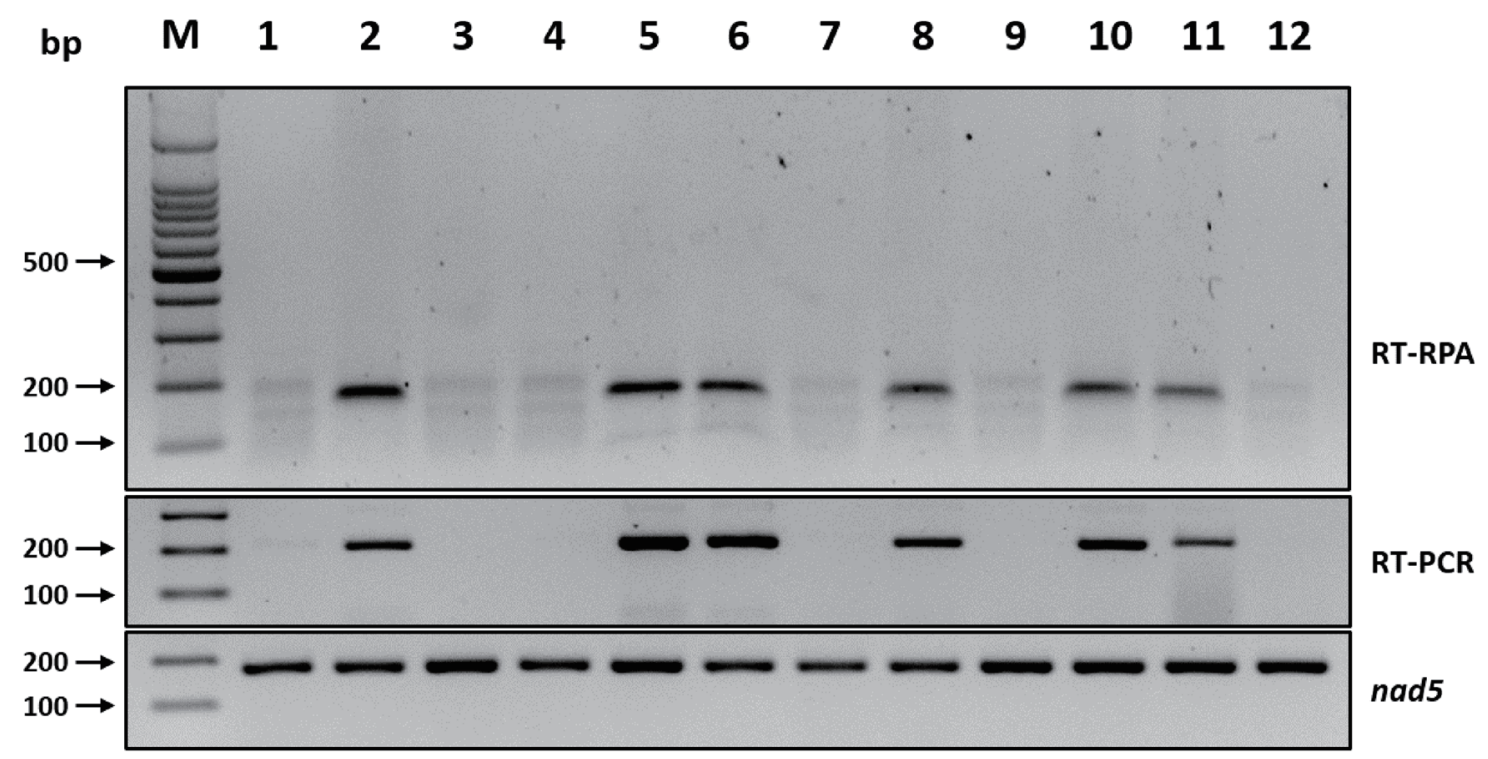

Fig. 4. Sample test of apple scar skin viroid (ASSVd) by reverse transcription recombinase polymerase amplification (RT-RPA). Lane M, 1,000bp marker; lanes 1-12, products amplified from 12 ASSVd symptom-like apple leaf samples. nad 5 was used as an internal control. RT-PCR, reverse transcription polymerase chain reaction.

and RT-RPA in six assayed apple leaf samples (Fig. 4), indicating that the RT-RPA could be used to detect ASSVd in fieldcollected samples.

ASSVd has become one of the most devastating viroids to affect pome production in Korea, and losses from ASSVd infection in Korea are expected to increase. Therefore, to ensure early detection, it is crucial to develop a rapid and accurate detection method. Currently, RT-PCR is widely used to screen pome fruits for ASSVd infection. However, the application of RT-PCR is limited by the method's reliance on specialized laboratory equipment, complex reaction conditions, and time-consuming procedures (Henson and French, 1993). A recent study reported that a 30-min RT-RPA assay was highly sensitive, with a detection limit of $10^{4}$ copies of ASSVd cDNA (Lee et al., 2018). RT-LAMP assays require at least four sets of primers and a relatively high reaction temperature. To overcome these inherent shortcomings, the present study developed a rapid, sensitive, specific, and reliable RT-RPA assay that requires only a single primer set, single-temperature incubation $\left(42^{\circ} \mathrm{C}\right)$, and relatively short reaction time (10 $\left.\mathrm{min}\right)$. The assay was 10 times more sensitive than RT-PCR, and no cross-reaction was observed for any of the other major apple viruses. Moreover, the established RT-RPA assay was also successfully applied to field-collected apple leaf samples.

To the best of my knowledge, this is the first report of an RTRPA assay for ASSVd detection. The assay can be utilized for viroid-free certification, for the in vitro screening of introduced tissue culture plants, and for ASSVd-resistance screening.

\section{Conflicts of Interest}

No potential conflict of interest relevant to this article was reported.

\section{Acknowledgments}

This work was carried out with the support of Cooperative Research Program for Agriculture Science and Technology Development (Project No. PJ014947032021) Rural Development Administration, Republic of Korea.

\section{Electronic Supplementary Material}

Supplementary materials are available at Research in Plant Disease website (http://www.online-rpd.org/).

\section{References}

Diener, T. O. 2001. The viroid: biological oddity or evolutionary fossil? Adv. Virus Res. 57: 137-184.

Hadidi, A., Huang, C., Hammond, R. W. and Hashimoto, J. 1990. Homology of the agent associated with dapple apple disease to 
apple scar skin viroid and molecular detection of these viroids. Phytopathology 80: 263-268.

Hammond, R. W. and Zhang, S. 2016. Development of a rapid diagnostic assay for the detection of tomato chlorotic dwarf viroid based on isothermal reverse-transcription-recombinase polymerase amplification. J. Virol. Methods 236: 62-67.

Hashimoto, J. and Koganezawa, H. 1987. Nucleotide sequence and secondary structure of apple scar skin viroid. Nucleic Acids Res. 15: 7045-7052.

Henson, J. M. and French, R. 1993. The polymerase chain reaction and plant disease diagnosis. Annu. Rev. Phytopathol. 31: 81-109.

Heo, S., Kim, H. R. and Lee, H. J. 2019. Development of a quantitative real-time nucleic acid sequence based amplification (NASBA) assay for early detection of apple scar skin viroid. Plant Pathol. J. 35: 164-171.

Higgins, M., Ravenhall, M., Ward, D., Phelan, J., Ibrahim, A., Forrest, M. S. et al. 2019. PrimedRPA: primer design for recombinase polymerase amplification assays. Bioinformatics 35: 682-684.

Khan, S., Mackay, J., Liefting, L. and Ward, L. 2015. Development of a duplex one-step RT-qPCR assay for the simultaneous detection of apple scar skin viroid and plant rna internal control. J. Virol. Methods 221: 100-105.

Kim, D.-H., Kim, H.-R., Heo, S., Kim, S.-H., Kim, M.-A., Shin, I.-S. et al. 2010. Occurrence of apple scar skin viroid and relative quantity analysis using real-time RT-PCR. Res. Plant Dis. 16: 247-253. (In Korean)

Kim, H. R., Lee, S. H., Lee, D. H., Kim, J. S. and Park, J. W. 2006. Trans- mission of apple scar skin viroid by grafting, using contaminated pruning equipment, and planting infected seeds. Plant Pathol. J. 22: 63-67.

Kim, N.-Y., Lee, H.-J., Kim, N.-K., Oh, J., Lee, S.-H., Kim, H. et al. 2019. Occurrence pattern of viral infection on pear in korea and genetic characterization of apple scar skin viroid isolates. Hortic. Sci. Technol. 37: 767-778.

Kumar, S., Singh, L., Ram, R., Zaidi, A. A. and Hallan, V. 2014. Simultaneous detection of major pome fruit viruses and a viroid. Indian J. Microbiol. 54: 203-210.

Lee, H.-J., Kim, H.-J., Lee, K. and Jeong, R.-D. 2020. Rapid detection of peach latent mosaic viroid by reverse transcription recombinase polymerase amplification. Mol. Cell Probes 53: 101627.

Lee, S. H., Ahn, G., Kim, M.-S., Jeong, O. C., Lee, J. H., Kwon, H. G. et al. 2018. Poly-adenine-coupled LAMP barcoding to detect apple scar skin viroid. ACS Comb. Sci. 20: 472-481.

Osaki, H., Kudo, A. and Ohtsu, Y. 1996. Japanese pear fruit dimple disease caused by apple scar skin viroid (ASSVd). Jpn. J. Phytopathol. 62: 379-385.

Panno, S., Matić, S., Tiberini, A., Caruso, A. G., Bella, P., Torta, L. et al. 2020. Loop mediated isothermal amplification: principles and applications in plant virology. Plants 9: 461.

Piepenburg, O., Williams, C. H., Stemple, D. L. and Armes, N. A. 2006. DNA detection using recombination proteins. PLoS Biol. 4: e204.

Walia, Y., Kumar, Y., Rana, T., Bhardwaj, P., Ram, R., Thakur, P. D. et al. 2009. Molecular characterization and variability analysis of apple scar skin viroid in india. J. Gen. Plant Pathol. 75: 307-311. 